\title{
Suggestions to improve outcomes for male victims of domestic abuse: a review of the literature
}

\author{
Tanis Moore ${ }^{1}$ (D)
}

Received: 23 November 2020 / Accepted: 6 September 2021 / Published online: 6 October 2021

(c) The Author(s) 2021

\begin{abstract}
Male victims of domestic abuse (DA) face a number of barriers to seeking help from their abusive relationships. Though available research has focussed primarily on exploring many of these challenges, few suggestions have been made on how to reduce or resolve them. It is necessary to establish a comprehensive plan to affect change at multiple levels in society in order to improve outcomes for this underserved population. This paper begins with a literature review examining in detail the many reasons why male victims of DA may refuse to seek help in an abusive relationship. Using the main key words, male victims combined with several common phrases related to the phenomenon of abuse including domestic abuse, domestic violence, and intimate partner violence, the review revealed several common reasons that male victims of DA refuse to seek help. These reasons include refusal or reluctance to view their experiences as abuse, hesitancy to identify with victimizing language, lack of available supportive services, embarrassment, shame, loss of masculinity, fear of being judged or disbelieved by others, fear of police response, and devotion to their family. Based on this review, a list of suggestions by the author is provided for changes that can be made to counter these barriers and improve male help-seeking. These suggestions are comprised of four broad themes: increasing public awareness, addressing the unique needs of male victims of DA, improving training for service providers, and increasing funding for services targeted to male victims of DA. A section exploring some of the unique concerns of gay, bisexual, and transgender men is included. International trends in the development and provision of services for male victims show that while increasing attention is being given to this vulnerable population, there are still significant gaps in available supports.
\end{abstract}

Keywords Male $\cdot$ Victims $\cdot$ Domestic $\cdot$ Abuse $\cdot$ Change

Tanis Moore

tanis.moore@hotmail.com

1 Canadian Association for Equality/Canadian Centre for Men and Families, Athabasca

University, Chestermere, Canada 


\section{Introduction}

Male victims of DA have historically been an under-served population and have received relatively little focus in research on intimate partner violence (Drijber et al. 2013; Laskey et al. 2019; Lysova et al. 2020b). Increasingly, researchers are directing their attention to the needs of male victims, especially in light of continued research that challenges the stereotypical view of domestic violence that casts men as perpetrators and women as victims (Neal and Edwards 2016; Ramsey 2015; Stemple et al. 2017; Straus 2011). It is important to state that this new focus is in no way intended to minimize the experiences of female victims, but rather to develop a more rounded and complex view of the subject that accounts for a diversity of experiences. For example, gendered assumptions of domestic abuse (a term chosen instead of domestic violence to reflect the diversity of abuse that is not physically violent in nature) not only preclude scenarios in which there is a female perpetrator of abuse or reciprocal abuse between both partners, but also fail to account for diverse couplings such as LGBT or nonmonogamous partnerships (Dixon et al. 2020; Donovan and Barnes 2019; Fisher and Pina 2013; Laskey et al. 2019). More importantly, gendered assumptions of DA neglect the experiences of male victims, making them more likely to ignore or minimize their experiences and less likely to reach out for assistance (Lysova et al. 2020a, b). Much of the current research on male victims of DA commonly focusses on these and other barriers that prevent these men from seeking support in their situations (Bates 2019; Huntley et al. 2019; Lysova et al. 2020b). Despite this, comparatively few suggestions have been made to address these barriers to male help-seeking. I aim to address this gap in this paper by first exploring the existing research in detail to compile a list of several reasons that male victims of DA may be reluctant to seek help. Based on these reasons, I offer suggestions on changes that can be made to help male victims of DA feel more comfortable reaching out for help, and how services and communities can become more accommodating to the unique needs of male victims. If changes can be made to address how male victims of DA are viewed and treated in society, I believe this will result in positive outcomes for this vulnerable population.

\section{Barriers to help seeking}

The goal of this literature review is to present a list of reasons why male victims of DA may be reluctant to seek assistance so that suggestions on how to challenge this problem can then be made based on these reasons. Furthermore, by exploring the reasons for hesitancy first, it allows us to better understand the extent to which change may involve targeted efforts towards male victims themselves or broader efforts to challenge attitudes in service providers or society at large. Because of this goal, the review was not guided by a particular theoretical framework, but rather a broad effort to try to seek as many perspectives as possible. 
Key terms such as male victims paired with terms such as domestic abuse, domestic violence, and intimate partner violence made up the bulk of phrases used to seek literature in peer reviewed journals. It is worth noting that despite specifically searching for they key phrase male victims, by far the majority of the articles encountered discussed concerns surrounding female victims of DA. As a result, much of the literature for this review came either from accessing citations from similar works, or from accessing bodies of work from authors experienced in the subject of male victims of DA. Finally, another source of information for this literature review came from the experiences of the workers and clients of the Canadian Centre for Men and Families. In asking some of these men about their experiences, this provided further ideas for suggestions on how to begin to seek change. Based on these sources of research, the following section explores several reasons why male victims of DA may be hesitant to seek help for their experiences.

\section{Refusal or reluctance to view their experiences as abuse}

Male victims of DA may simply not feel as though the negative behaviours they face in their relationships constitute abuse (Lysova and Dim 2020; Tsang 2015; Walker et al. 2020). There may be a number of contributing factors to this. As already mentioned, there is a prevailing narrative that domestic violence takes on a particular form, most notably that of a male perpetrator abusing a female victim (Ramsey 2015; Walker et al. 2020). Women are more likely to report their abuse to police, and police statistics demonstrate that women are more likely to be at risk for serious harm or death (Dixon et al. 2020). This tends to inform decision making about the need for services and for future research into the problem of female victimization, which further bolsters the idea that DA primarily impacts women (Laskey et al. 2019). Furthermore, the Duluth Model, the most commonly used police intervention model in the United States and Canada, operates under a highly gendered perspective (Bohall et al. 2016). Training for counsellors and DA crisis line workers often includes similar gendered influences as well (Douglas and Hines 2011; Hogan et al. 2012). Gendered philosophies such as patriarchy theory ascribe a certain level of power to men which may predispose service providers to assumptions about men's status as perpetrators rather than victims of DA (Lysova et al. 2020a). For example, the Duluth Model, rooted primarily in ideological and political frameworks rather than in evidence based practice, suggests that male batterers use DA as a means of demonstrating power and control over their female partners (Corvo et al. 2009). It should come as little surprise that a model that assumes such narrow motivations for DA would fail to capture an accurate picture of the experience of male victims, many of whom often suffer abuse from female partners (Bohall et al. 2016; Douglass et al. 2020). That such a model is mainstream in both the legal system and support services not only leaves many workers without effective guidelines on how to respond to or support male victims of DA, but also contributes to the view that male victims of DA are elusive in comparison to female victims (Bohall et al. 2016; Douglas and Hines 2011; Lysova et al. 2020a). This view is also reflected in general 
society, where female-perpetrated DA is often minimized and men are frequently blamed for their own victimization (Lysova and Dim 2020; Tsang 2015; Walker et al. 2020). This view is common across cultures but may be even more pronounced in cultures with more traditional views of gender roles or rigid expectations of masculinity (Ayodele 2017; Cheung et al. 2009; Thobejane and Luthada 2019). As a result of the pervasiveness of dismissive attitudes towards male victimization, men may be less inclined to view all but the most serious and violent acts from their partner as abuse (Arnocky and Vaillancourt 2014; Lysova and Dim 2020; Lysova et al. 2020b).

\section{Hesitancy or unwillingness to identify with victimizing language}

The language associated with domestic abuse is a sticking point for many male victims. When characterizing abusive experiences using terms such as domestic violence, men may feel disinclined to believe that their experiences align with such terms (Lysova et al. 2020a, 2020b). This is potentially because men may be more likely to experience abuse from their female partners that does not constitute physical violence (Dim and Elabor-Idemudia 2018; Huntley et al. 2019). However, it may also be due in part to the still-common belief that women make up the majority of victims of DA, despite national surveys and formal research showing equal and even higher levels of male victimization (Hogan et al. 2012; Laskey et al. 2019; Lysova et al. 2020b; Neal and Edwards 2016). Although the feminist and women's shelter movements were instrumental in bringing attention to and developing services for domestic violence victims, the sole focus on female victims resulted in a politicizing of the word violence (Barner and Carney 2011; Walker et al. 2020). One such example is the idea that domestic violence is an act of patriarchal power meant to exercise control over female victims (Barner and Carney 2011; Douglass et al. 2020; Dutton 2012). As a result, men who experience DA, even those who experience physical violence, may prefer not to associate their experiences with the term domestic violence (Bates 2019; Tsang 2015; Walker et al. 2020). Although slightly more neutral, terms like domestic abuse may still fail to ring true with many male victims, especially with men who are inclined to make excuses or justifications for their partners' behaviours (Dixon et al. 2020).

Furthermore, men who struggle with DA may also be reluctant to identify as victims (Bates 2019; Dixon et al. 2020; Walker et al. 2020). This is due in part to the narrative reasons previously discussed surrounding female victims and male perpetrators (Ramsey 2015; Walker et al. 2020). However, traditional masculine norms focus on male agency and control, and these notions are at odds with the idea of being a victim (Bates 2019; Lysova and Dim 2020; Walker et al. 2020). In some ways, denial of this victimization language may come from a desire to avoid appearing vulnerable, both internally and to others (Ayodele 2017; Lysova and Dim 2020). Men may avoid this sense of vulnerability as a coping mechanism to help maintain their self-esteem and to combat shame, depression, and anxiety (Dim and ElaborIdemudia 2018; Pešáková 2013; Shorey et al. 2011). Alternatively, avoiding a victim label may mean preserving an appearance of masculinity to others, especially in 
cultures with more rigid role expectations for men (Ayodele; 2017; Thobejane and Luthada 2019).

\section{Isolation and lack of supportive services}

Male victims of DA may feel that they are alone and no one else understands their experiences (Bates 2019; Javaid 2017; Lysova et al. 2020b). Feeling as though no other men are experiencing what they are experiencing may lead male victims of DA to feel embarrassed or ashamed to tell anyone about their victimization (Bates 2019; Dixon et al. 2020; Lysova et al. 2020b). Men who feel isolated in their relationship may feel that it is safer or easier to stay with their partner, or that they do not have adequate social supports to attempt to leave (Dixon et al. 2020; Machado et al. 2017). These men may also struggle with depression, anxiety, and diminished self-confidence, which may negatively impact their help-seeking behaviours (Huntley et al. 2019). On a broader level, male victims may believe that there are no resources available to help them, or that the available DA resources are targeted only toward female victims (Huntley et al. 2019; Machado et al. 2017). Despite this, men who experience high-severity DA may feel more inclined to try to reach out to these services even if they doubt that they will offer a fully supportive response (Douglas et al. 2012; Lysova et al. 2020a; Machado et al. 2017). Men who attempt to reach out to DA resources and receive an unsupportive response may be less likely to seek help in future incidents of DA (Douglas and Hines 2011; Douglas et al. 2012).

\section{Embarrassment, shame, and loss of masculinity}

Male victims may not want to seek help for their victimization because they feel it would reflect poorly on their masculinity (Huntley et al. 2019; Pešáková 2013). For many men, being a victim of DA, especially at the hands of a female partner, is inconsistent with male gender roles (Arnocky and Vaillancourt 2014). Individual men may experience these gender roles differently, but common descriptions include being self-reliant, stoic, strong, dominant, autonomous, and being in control of one's life (Bates 2019; Dixon et al. 2020; Thobejane and Luthada 2019). Men who hold these views of masculinity may avoid seeking help for various reasons. Some men may feel that it goes against their idea of stoicism and self-reliance to get help from others rather than trying to manage the problem themselves (Dixon et al 2020). Others may feel that to leave and seek outside assistance would reflect poorly on their ability to act as a provider for their family, especially if children are involved (Ayodele 2017; Lysova et al 2020b; Tsang 2015). Still others may fear looking weak or being labelled a 'wimp', a 'coward', or 'feminine' (Arnocky and Vaillancourt 2014; Douglas \& Hines 2011; McCarrick et al. 2016; Tsui et al. 2010). Fear of emasculation in this manner may have different significance in different cultures (Cheung et al. 2009; Thobejane \& Luthada 2019). For some men, a challenge to their masculinity may simply represent a blow to their self-confidence, pride, or personal image (Bates 2019; Huntley et al. 2019; Tsang 2015). For other men, however, it may have broader implications not only in their personal view of themselves, but in how 
their extended families, friends, and communities see them as well (Ayodele 2017; Thobejane and Luthada 2019).

\section{Fear of being judged, stereotyped, or disbelieved by others}

Male victims of DA may avoid seeking help because they fear that others will judge or stereotype them (Bates 2019; Lysova et al. 2020b). Eckstein and Cherry (2015) conducted research that solicited the opinions of nearly 2000 men and women using a hypothetical male victim of a female abuser. In this research, the most common category of response was blaming, in which participants described the male victim as having a passive personality, being weak-minded or less assertive, and of having no backbone (Eckstein and Cherry 2015). Men may fear that others will view them as 'pathetic' or wonder why they were 'putting up with' the abuse (Bates 2019). Minimizing the severity of the abuse and victim-blaming have been reported as common responses to male victims' disclosures (McCarrick et al. 2016; Walker et al. 2020). Some cultures may be less tolerant of male victims of DA than others, and in these cultures publicly admitting one's victimization, let alone asking for help, could lead to serious social stigmatization (Cheung et al. 2009; Ayodele 2017).

Another concern male victims of DA involves not being believed by others about their victimization (Bates 2019). This may be due in part to the social narrative that views men as perpetrators rather than victims (Ramsay 2015). However, it may also be due to cultural views of masculinity, especially views of strength and dominance, influencing individuals' views on how men should be 'expected' to respond to DA (Bates 2019; Huntley et al. 2019; Walker et al. 2020). Whatever the reason, disbelief is a common response to male victims' disclosures, not only from the public but also from DA services and counsellors as well (Douglas and Hines 2011; Hogan et al. 2012; McCarrick et al. 2016). Research by Douglas and Hines (2011) found that many men who reached out to DA hotlines or agencies experienced a range of negative responses including being told those agencies only help women, being ignored, being treated as the batterer, and being referred to batterers' resources. Similarly, common responses from counsellors may involve surprise, taking male victims' claims less seriously, doubting their stories, and assuming that they are actually the batterer (Hogan et al. 2012; Perryman and Appleton 2016). Not surprisingly, male victims who experience this kind of treatment will be more likely to rate such services unfavourably (Douglas et al. 2012). They may also be less likely to reach out to these and similar services in the future, which may exacerbate feelings of helplessness, isolation, and that they have nowhere to go for support (Lysova et al. 2020b).

\section{Fear of police response}

Although law enforcement may respond to male victims of DA in many of the same ways that other DA related services may respond, there are concerns unique to contacting police that may prevent men from reaching out to them (Lysova et al. 2020a; McCarrick et al. 2016). First, police officers may behave in more dismissive or hostile ways than formal services like counsellors, laughing at male victims' claims or 
even outright insulting male victims (Lysova et al. 2020a). The police may simply refuse to assist in the situation or claim that there is nothing that they would be able to do (Drijber et al. 2013). However, a more frightening prospect may involve not only being treated as the abuser but being arrested (Lysova et al. 2020a; McCarrick et al. 2016). Feminist domestic violence models and policies based on gendered assumptions may increase the likelihood that male victims are arrested (Barner and Carney 2011; Bohall et al. 2016; Lysova et al. 2020a). A particularly damaging form of abuse from female perpetrators involves falsely claiming that their male victim is actually the abuser, and this is likely to further increase the risk of the man being arrested rather than helped (Walker et al. 2020). Men are more likely to attempt to reach out to the police if they have been severely injured because of the physical evidence to demonstrate their victimization (Douglas et al. 2012; Drijber et al. 2013). However, fear of arrest and even a violent altercation with the police may hinder male victims from reaching out in all but the most serious of cases (Drijber et al. 2013; Lysova et al. 2020a).

\section{Dedication to spouse or family}

A common reason cited in the literature for male victims' refusal to seek outside help is their dedication to their family (Huntley et al. 2019). Many male victims expressed that they did not want their relationship with their partner to end, only that they wanted the abuse to stop (Huntley et al. 2019; Lysova et al. 2020b). Some men cited the importance of love and their marriage vows to their partners and expressed concern that seeking help would be tantamount to leaving their spouse (Bates 2019; Lysova et al. 2020b). Some men may feel concerned for their partners and may prefer to stay to try to address the matter themselves (Lysova et al. 2020b). This problem-solving behaviour may involve calming their spouse down, changing their own behaviour to try to appease their spouse, or attempting to fix the problem they believe is causing their partner's anger (Lysova et al. 2020b; Machado et al. 2017; Pešáková 2013). On the other hand, some men may feel afraid to leave their spouse for fear that she may retaliate using the police or court systems in a form of abuse known as legal and administrative partner aggression (Berger et al. 2015; Lysova et al. 2020b; Walker et al. 2020). Berger et al. (2015) examined this form of abuse in detail and provided such examples as making false accusations of abuse to the police, contacting child protection services, and attempting to wrest custody of the children away from the abused partner.

Having children with their abusive partner sparks several other reasons why men may refuse to seek help (Lysova et al. 2020b; Machado et al. 2017; Pešáková 2013). Men may believe that they are protecting their children by staying in the relationship (Lysova et al. 2020b). This may be out of a desire to keep the abuse focussed on them instead of the children (Machado et al. 2017; Lysova et al. 2020b). Male victims may also feel that if they stay in the abusive relationship, they will be able to better provide for their children (Lysova et al. 2020b). This is especially true in cultures that look poorly upon male victims of DA (Ayodele 2017; Tsang 2015). Finally, as previously stated, male victims may fear losing access to their children 
entirely if they report the abuse (Bates 2019; Berger et al. 2015; Walker et al. 2020). This may be due to being arrested by the police due to a false accusation or being forced out of their children's lives due to the abuser trying to gain full custody or filing a protection order (Berger et al. 2015). This form of abuse is particularly effective due to law enforcement and legal biases and assumptions that men are more likely to be perpetrators than victims (Berger et al. 2015; Lysova, et al. 2020a).

\section{Suggestions for change}

My suggestions to improve outcomes for male victims of DA can be broken down into four broad themes: increasing public awareness, addressing the unique needs of male victims of DA, improving training for service providers, and increasing funding for services targeted to male victims of DA. Each of these will be considered in turn.

\section{Increasing public awareness}

As dismissive attitudes towards male victims of DA run deeply throughout formal services and the public alike, a broad-scale public awareness campaign could help draw attention and add legitimacy to the problem of male victimization (Bates 2019; Hogan et al. 2012; Lysova et al. 2020a; McCarrick et al. 2016; Tsui et al. 2010). A comprehensive strategy to bring awareness to male victims of DA includes challenging the current narrative around DA (Ramsey 2015). Such a campaign may take a number of forms. For example, television and public advertisements could be used not only to highlight the fact that men suffer from DA as well as women, but they could also directly challenge stereotypes and misinformation and correct the common view that women are more frequently victimized than men (Neal and Edwards 2016; Straus 2011). Naturally, care must be taken to ensure this message is not presented in a way that minimizes the traumatic experiences of female victims of DA, but that simply demonstrates the more complete picture of what abuse looks like. Similarly, classes or public service announcements in high schools, colleges, and universities addressing the problem of male victimization could help to target common views that maintain current narratives about DA (Fisher and Pina 2013; Tsui et al. 2010). These narratives may include stereotypes about the male gender role or male rape myths such as the idea that "A man can enjoy sex even if it is being forced on him" (Fisher and Pina 2013, p. 57). Views that discount women as perpetrators, such as the notion that women are passive, weak, and gentle, could also be addressed (Douglass et al. 2020). Such benevolent sexism not only undermines the significance of male victimization, but also minimizes the agency of women and fails to address the complexity of why women also engage in DA (Douglass et al. 2020).

Similar public service announcements could help the public recognize when male victims are in need by drawing attention to the different ways that men and women might behave when involved in an abusive relationship (Machado et al. 2017). If 
men believe that people will recognize and be sympathetic to their distress, this may improve their desire to reach out for help (Lysova et al. 2020b; Perryman and Appleton 2016). Furthermore, this kind of messaging could also be helpful to men who do not recognize that they are in abusive relationships (Dixon et al. 2020; Huntley et al. 2019). Whether men believe that the behaviour they are experiencing is normal or they simply minimize or excuse the abuse, bringing awareness to behaviours that constitute abuse could help men acknowledge their situation and take action (Arnocky and Vaillancourt 2014; Walker et al. 2020). Further research into male victims of DA could help to identify gaps in knowledge surrounding male victimization. This may include determining specific differences in the ways men and women respond to and recover from DA (Laskey et al. 2019; Spencer et al. 2019). Such research may inform the development of services targeted to male victims, which may then lend further legitimacy to and awareness of their experiences (Tsang 2015; Tsui et al. 2010).

\section{Addressing the unique needs of male victims of DA}

DA can be a traumatic experience with serious consequences for both men and women, and many of these consequences may be similarly experienced by both sexes (Laskey et al. 2019; Spencer et al. 2019). However, just as it is important to understand the unique ways female victims experience DA, understanding the unique experiences of men and the barriers they face to seeking help is important to improving their outcomes (Lysova et al. 2020b). First, it is important to know what kinds of services male victims of DA might be most likely to find helpful (Douglas et al. 2012). Knowing this involves understanding the kinds of DA that men may be most likely to experience (Dim and Elabor-Idemudia 2018). Research has demonstrated that men experience a variety of DA including physical and sexual abuse of varying severity, but men may more commonly experience indirect, non-physical abuse such as psychological abuse or abuse of legal and administrative systems (e.g., law enforcement or child protection services) during separation (Berger et al. 2015; Dim and Elabor-Idemudia 2018; Lysova and Dim 2020; Walker et al. 2020). Many men, especially those who do not wish to leave their families for various reasons, will likely not want to use a DA shelter, and may hesitate to contact police or legal services (Huntley et al. 2019; Lysova et al. 2020b). As negative mental health outcomes are a common experience across multiple different types of DA, counselling and other support services provided by individuals who are well versed and specifically trained in men's victimization could prove particularly helpful (Hogan et al. 2012; Tsui et al. 2010).

Some specific actions may be taken to improve the likelihood that male victims will recognize their abuse and reach out for help in the first place. As domestic violence is often a charged term that may not accurately describe much of the victimization men face, use of more neutral and all-encompassing language such as domestic abuse, partner abuse, or relationship boundary crossings may help men to feel more comfortable opening up about their experiences (Bates 2019; Walker et al. 2020). On a similar note, use of language such as survivor rather than victim 
may resonate more positively with men who have experienced DA (Lysova and Dim 2020). Creation of screening tools specifically targeted to male victims may help increase male disclosure (Drijber et al. 2013; Perryman and Appleton 2016). Rather than asking specifically about abuse or violent behaviour, these screening tools may simply involve checklists of behaviours or Likert scales assessing threat perceptions (Arnocky and Vaillancourt 2014). If men report experiencing behaviours that constitute DA, they should be validated and asked how they would like to proceed (Walker et al. 2020). Men may have many reasons they would prefer to stay rather than attempt to leave or to contact police (Lysova et al. 2020b). Since men often hold their personal agency in such high regard, it may help them to feel better if they can regain or maintain a sense of control about how they manage their situation (Dixon et al. 2020; Huntley et al. 2019).

In fact, promoting agency and providing validation are potentially two of the most important ways to combat the kinds of negative consequences that male victims of DA face (Dixon et al. 2020). Male victims of DA may suffer from multiple adverse effects both during and following the abuse, including depression, anxiety, low self-esteem, shame, social isolation, post-traumatic stress disorder, substance abuse, and suicidal ideation (Pešáková 2013; Randle and Graham 2011; Walker et al. 2020). Although men and women both experience a number of negative effects as a result of DA, being seen as a victim often strikes at the very core of a man's sense of self in a way that is unique (Randle and Graham 2011; Tsang 2015; Walker et al. 2020). Male gender roles typically emphasize agency, self-reliance, and stoicism, so male victims of DA may find that they not only experience the adverse effects already mentioned but they may also feel divested from a key part of their identity (Ayodele 2017; Bates 2019). An important part of combatting this loss of self is in reassuring male victims of their agency and autonomy (Dixon et al. 2020). One helpful way this can be achieved is to reframe vulnerability as strength, and a desire to seek change and assert one's needs as agency instead of weakness (Englar-Carlson and Kiselica 2013).

Another important focus in improving outcomes for male victims is in providing a great deal of validation (Dixon et al. 2020; Englar-Carlson and Kiselica 2013). The negative mental health outcomes male victims experience may occur specifically in response to the abuse; however, they may also find these effects compounded by external responses to their victimization (Walker et al. 2020). Being faced with minimizing or victim-blaming behaviour from their community is a common difficulty (Bates 2019). However, being portrayed as a perpetrator by DA agencies or police can be especially traumatizing, especially if such a role reversal results in arrest (Lysova et al. 2020b). As a result, male victims of DA have often spent an extended period of their lives struggling with self-doubt, and doubt from those around them (Dixon et al. 2020; Huntley et al., 2020; Walker et al. 2020). Providing a place where these men can find genuine validation and feel safe discussing their experiences without fear of social reprisal could make a significant difference to mental health outcomes following DA (Dixon et al. 2020; Hogan et al., 2020). Group counselling settings with other men who have had similar experiences may be an especially helpful format that could help male victims feel less isolated (Dixon et al. 2020). 
Male victims of DA involved in the legal system with their partners may require special considerations depending on their goals (Berger et al. 2015). For example, men who have contacted the police after an incident of DA may not necessarily want the relationship to end (Huntley et al. 2019; Lysova and Dim 2020). In this case, couples counselling, especially targeted to DA perpetrators, could be helpful in addressing the problems maintaining the abuse (Spencer et al. 2019). Additionally, an assigned social worker could help with assessing risk of further abuse and could educate the family in healthy relational behaviours (Amundson and Lux 2016). Alternatively, some men may want to leave the relationship but may feel unable to because they worry about losing access to their children (Lysova et al. 2020b). In cases where it is appropriate, mediation, parenting coordination, and legal advice could be offered to help ease the transition out of the relationship (Amundson and Lux 2016). However, as in all other service provision, it is important that those involved in a legal or administrative battle behave in a neutral manner to both parties (Berger et al. 2015). A neutral legal system is necessary to ensure fair outcomes, especially when false accusations may be used as yet another tool of abuse (Berger et al. 2015; Lysova et al. 2020a).

\section{Improving training for service providers}

Training for police and service providers should approach the subject of DA using a gender-neutral lens (Walker et al. 2020). Ideally, this training should be informed by up-to-date research that addresses the complexities of DA and challenges assumptions (Du Mont et al. 2013; Hogan et al. 2012; Perryman and Appleton 2016). Crisis line workers and other similar support providers should be trained to recognize that abuse is possible against both men and women (Walker et al. 2020). Furthermore, care should be taken to ensure that male victims are not given referrals to batterers programs when reaching out for help (Douglas and Hines 2011). In training and research discussing DA, outdated perspectives that focus solely on male perpetration should be replaced with more gender-inclusive ideas such as social learning theory, the contribution of alcohol or other drugs, and the use of DA as a form of maladaptive conflict resolution (Bohall et al. 2016; Dixon et al. 2020; Douglass et al. 2020; Dutton 2012; Spencer et al. 2019). Similarly, DA models that promote one-sided views of abuse and violence such as the Duluth Model should be phased out of mainstream favour in support services and the legal system as these models not only minimize and cast doubt on the experiences of male victims, but also fail to account for the experiences of abuse of those in same sex or polyamorous relationships (Bohall et al. 2016; Donovan and Barnes 2019). Training for police and other service providers should acknowledge that DA cases are commonly symmetrical and that women are indeed capable of violent and abusive behaviour (Douglass et al. 2020; Neal and Edwards 2016). Myths about male victimization should be addressed in training as well (Fisher and Pina 2013; Javaid 2017).

Ideally, when reports are made to police, both partners' stories should be considered and both should be treated with equal respect and dignity for the duration of 
the investigation (Bates 2019; McCarrick et al. 2016). Service providers should, as much as possible, attempt to support men based on their needs rather than based on assumptions about victims of DA (Hogan et al. 2012). For example, service providers should use the language that men feel comfortable with. That is, if they do not identify as victims, service providers should avoid using victim language (Lysova and Dim 2020). Another common concern of male victims of DA is how male gender roles impact them, the way they behave, and the way they see themselves in the wake of the abuse (Arnocky and Vaillancourt 2014). If service providers engage in conversation with men about masculinity, use of the phrase 'toxic masculinity' should be avoided as many men find this to be an insulting term (Barry et al. 2020). Instead, conversations should be framed around positive masculinity and how men conceptualize masculinity in their lives (Barry et al. 2020; Englar-Carlson and Kiselica 2013). Counselling should address men's experiences of being stereotyped in their communities and offer personal strategies to help them ignore or combat such experiences (Hogan et al. 2012; Perryman and Appleton 2016). Above all, it is critically important that service providers and police refrain from responding to male victims of DA with doubt, dismissiveness, or incredulity (Hogan et al. 2012; Machado et al. 2017).

\section{Increasing funding for services targeted to male victims of DA}

Fortunately, in many cases, female victims of DA will have multiple agencies available to help them that are tailored to their needs (Douglas and Hines 2011; Douglas et al. 2012). Ideally, services aimed specifically at men should be available in the same way female-only services are available, at least in proportion to the volume of need within a given area (Tsui et al. 2010). In conducting research into male and female victims of DA, it is important that data on the numbers of each group be collected using multiple sources including police and crime data, research studies, and national surveys (Dixon et al. 2020; Lysova et al. 2020b). Reliance only on police report numbers will likely yield results that do not reflect the actual numbers of male victims, given their frequent reluctance to report their experiences (Lysova et al. 2019; Lysova et al. 2020b). Further research on the needs of male victims of DA may then be used to demonstrate the need for services as well as seek funding to help cover expenses (Tsui et al. 2010).

\section{Special considerations for gay, bisexual, and transgender men}

Specialized services for the unique needs of gay, bisexual, and transgender (GBT) individuals are rare, ill-funded, and do not receive a significant amount of attention in the wider community (Donovan and Barnes 2019). As previously discussed, models that assume a male perpetrator and female victim narrative will insufficiently describe the experiences of GBT men. (Bohall et al. 2016; Donovan and Barnes 2019; Ramsey 2015). However, due to the common stereotype of DA perpetrators being male and reluctance to accept the seriousness 
of female violence, male victims of male perpetrators may in some ways be treated with more legitimacy as 'victims' than male victims of female perpetrators (Douglass et al. 2020; Ramsey 2015; Stemple et al. 2017). In cultures that are intolerant of homosexuality, however, GBT male victims of DA may find it especially difficult, if not impossible, to seek help for fear of additional negative social ramifications (Thobejane and Luthada 2019). Due to a lack of supportive services in the community, GBT men may be more likely to turn to drug use to cope with DA (Bacchus et al. 2016). They may also be more likely to experience problems with their sexual health on account of not feeling comfortable disclosing their experiences to health practitioners or police (Bacchus et al. 2016; Javaid 2017). Finally, transgender men may experience unique forms of DA related specifically to their gender identities, such as their partner withholding access to hormones or medical appointments, which may make it especially difficult for them to access help (Laskey et al. 2019). Addressing the needs of male victims of DA necessitates understanding the different dynamics that may accompany diverse relationships.

\section{Potential barriers to implementing changes}

There are several barriers that may stand in the way of implementing some of the changes outlined in this literature review. First, there is the possibility that current gender and ideological paradigms may result in reluctance to acknowledge the commonality or severity of male victimization (Bohall et al. 2016; Hall 2012; Lysova et al. 2019). This is especially true if increasing focus on male victims is viewed as minimizing or competing for focus on female victims (Hall 2012). Bias in the social sciences towards female victims of DA may not only result in a failure to reach a consensus about the kinds of programs and services needed to support male victims, but it may result in research approaches that are dedicated more towards preserving the current status quo rather than seeking the truth (Dutton 2010; Hall 2012). Another potential barrier lies in the frequent reliance on police data to reflect the spectrum of DA (Lysova et al. 2019). Because many male victims are reluctant to report their victimization to police, reliance on such data for studies and for the development of programs may lead to the inaccurate belief that fewer resources are needed for this population (Hall 2012; Lysova et al. 2019). Further, the lack of services available for male victims of DA may then result in fewer men reaching out for help, resulting in fewer men being reflected in police data, which then results in a circular problem (Lysova et al. 2020b). As long as governments and broader global agencies such as the World Health Organization maintain that DA is primarily a problem perpetrated by men against women, funding and broader public support for many of the suggested changes in this paper may be more difficult to obtain (World Health Organization 2012). 


\section{International trends}

Cheung et al. (2009) outlined several agencies within Canada, the United States, and the United Kingdom that are specifically targeted to male victims of DA as opposed to male perpetrators. Tragically, the owner of one of the listed agencies, the Men's Alternative Safe House, committed suicide citing a lack of government funding to maintain the program (Cheung et al. 2009; Gerson 2013). More recently, another agency in Canada, the Canadian Association for Equality/ Canadian Centre for Men and Families (2021), found success in securing funding and has now been operating a family shelter for male victims of DA and their children since April of 2021. In the entirety of the U.S., there are believed to be only two shelters that focus on male victims of DA (New York Post 2017). Further, the U.S. National Domestic Violence Hotline (2021) has a page on its website in support of male victims of DA; however, elsewhere on the website the Duluth Model is posted prominently. In the U.K., the Mankind Initiative (2021), a hotline dedicated to male victims, states on its website that they are funded solely by the public and receive no government grants. A New South Wales Government (2020) website for Australian services for male victims of DV lists support phone lines men can call; however, only one, MensLine Australia, appears to be targeted towards male victims in specific, while 1800RESPECT is a unisex service and Men's Referral Service is a line targeted to male perpetrators. Internet searches for male victims' supports in multiple other countries revealed very few other targeted services. In many countries, this lack of services may be impacted largely by cultural expectations of men that largely preclude them being viewed as victims (Ayodele 2017; Cheung et al.2009; Thobejane and Luthada 2019). These search results illustrate the dearth of services aimed exclusively at male victims of DA as well as the common lack of government willingness to fund such supports. Positively, however, there were many articles indicating a need to address the concerns of this group, demonstrating an increasing awareness of their struggles.

\section{Considerations for future research}

Potential future research in this area might include specific methods of challenging stereotyped views in the community about male victims of DA. It is possible that these methods of challenging stereotypes will be differently effective amongst different cultural groups (Ayodele 2017; Cheung et al. 2009). Different approaches may be required depending on the extent of any culture's rigidity towards gender roles or expectations of masculinity (Ayodele 2017; Thobejane and Luthada 2019). Additionally, much of the research on this topic has explored the barriers that prevent men from reaching out for help in DA situations (Lysova et al. 2020b), but little research has been conducted to explore what conditions would make male victims feel more comfortable reaching out. Surveys soliciting 
affirmative responses as to what male victims would like to see in order to feel comfortable seeking help could provide further ideas on changes to make to improve outcomes in this area. Research directly comparing and contrasting the needs of male victims and female victims of DA may yield differences between the groups that produce helpful suggestions and further ideas for service provision. Further exploration on the different experiences of GBT men could similarly lead to increased awareness about their unique needs in this area.

\section{Conclusion}

DA is a pervasive problem that negatively impacts men, women, and children alike. Unfortunately, male victims still face a number of barriers to seeking help. Many of these challenges have been explored in some depth in the literature (Huntley et al. 2019). Throughout the literature, there have been suggestions about addressing some of these problems. However, I believe it is necessary to address these obstacles both at the level of service provision and at a societal level. In this paper, I have outlined a comprehensive list of changes that can be made to improve outcomes for male victims of DA in a variety of ways. These are changes that are not likely to happen overnight. Societal attitudes often take many years to shift. Even for female victims of DA, it took decades to address the seriousness of their struggles and lay down the foundation for the protections they now have in place today (Barner and Carney 2011). However, it is encouraging seeing the progress that has been made to improve outcomes for these vulnerable women. Knowing that male victims of DA struggle in many of the same ways, it is time that we address their concerns with the same urgency, care, and attention.

Acknowledgements I would like to express my sincere appreciation to my professor Dr. Hillary Sharpe for providing feedback and suggestions during the initial drafting of this paper, as well as for encouraging me to submit the paper for publication. I would not have come this far were it not for your kind assistance. Additionally, I would like to thank the members, volunteers, and service users of the Canadian Association for Equality/Canadian Centre for Men and Families for their continued support of my work. In particular, I would like to thank Michael Healey, who spent a great deal of time talking with me about common experiences of male victims of DA he encounters in his own work with these populations.

Data availability This manuscript has no associated data.

\section{Declarations}

Conflict of interest The corresponding author states that there is no conflict of interest.

Open Access This article is licensed under a Creative Commons Attribution 4.0 International License, which permits use, sharing, adaptation, distribution and reproduction in any medium or format, as long as you give appropriate credit to the original author(s) and the source, provide a link to the Creative Commons licence, and indicate if changes were made. The images or other third party material in this article are included in the article's Creative Commons licence, unless indicated otherwise in a credit line to the material. If material is not included in the article's Creative Commons licence and your intended use is not permitted by statutory regulation or exceeds the permitted use, you will need to obtain permission 
directly from the copyright holder. To view a copy of this licence, visit http://creativecommons.org/licen ses/by/4.0/.

\section{References}

Amundson JK, Lux GM (2016) Risk management in high-conflict divorce/parenting referrals: It's how you walk through the fire. Can J Counsell Psychother 50(3):10-22

Arnocky S, Vaillancourt T (2014) Sex differences in response to victimization by an intimate partner: more stigmatization and less help-seeking among males. J Aggress Maltreatment Trauma 23(7):705-724. https://doi.org/10.1080/10926771.2014.933465

Ayodele JO (2017) The socio-cultural causes of male victimization in domestic contexts in Lagos, Nigeria: a qualitative analysis. Int J Crim Justice Sci 12(2):252-269. https://doi.org/10.5281/zenodo. 1034670

Bacchus LJ, Buller AM, Ferrari G, Peters TJ, Devries K, Sethi G, White J, Hester M, Feder GS (2016) Occurrence and impact of domestic violence and abuse in gay and bisexual men: a cross sectional survey. Int J STD AIDS. https://doi.org/10.1177/0956462415622886

Barner JR, Carney MM (2011) Interventions for intimate partner violence: a historical review. Journal of Family Violence 26(3):235-244. https://doi.org/10.1007/s10896-011-9359-3

Barry J, Walker R, Liddon L, Seager M (2020) Reactions to contemporary narratives about masculinity: a pilot study. Psychreg J Psychol 4(2):8-21

Bates E (2019) "No one would ever believe me": an exploration of the impact of intimate partner violence victimization on men. Psychol Men Masc 1-38

Berger JL, Douglas EM, Hines DA (2015) The mental health of male victims and their children affected by legal and administrative partner aggression. Aggressive Behav 42(4):346-361. https://doi.org/10.1002/ab.21630

Bohall G, Bautista M, Musson S (2016) Intimate partner violence and the Duluth Model: an examination of the model and recommendations for future research and practice. J Fam Violence 31:1029-1033. https://doi.org/10.1007/s10896-016-9888-x

Canadian Centre for Men and Families (2021). First Family Shelter for Fathers and Children to Open in Toronto this April 2021. Retrieved from https://menandfamilies.org/familyshelter/

Cheung M, Leung P, Tsui V (2009) Asian male domestic violence victims: services exclusive for men. J Fam Violence 24:447-462. https://doi.org/10.1007/s10896-009-9240-9

Corvo K, Dutton D, Chen W (2009) Do Duluth Model interventions with perpetrators of domestic violence violate mental health professional ethics? Ethics Behav 19(4):323-340. https://doi.org/ 10.1080/10508420903035323

Dim EE, Elabor-Idemudia P (2018) Prevalence and predictors of psychological violence against male victims in intimate relationships in Canada. J Aggress Maltreatment Trauma 27(8):846-866. https://doi.org/10.1080/10926771.2017.1382638

Dixon L, Tretharne GJ, Celi EM, Hines DA, Lysova AV, Douglas EM (2020) Examining men's experiences of abuse from a female intimate partner in four English-speaking countries. J Interpers Violence. https://doi.org/10.1177/0886260520922342

Donovan C, Barnes R (2019) Help-seeking among lesbian, gay, bisexual and/or transgender victims/ survivors of domestic violence and abuse: the impacts of cisgendered heteronormativity and invisibility. J Sociol. https://doi.org/10.1177/1440783319882088

Douglas EM, Hines DA (2011) The helpseeking experiences of men who sustain intimate partner violence: an overlooked population and implications for practice. Journal of Family Violence 26(6):473-485. https://doi.org/10.1007/s10896-011-9382-4

Douglas EM, Hines DA, McCarthy SC (2012) Men who sustain violence from their female partners: Predictors of where they seek help and how they rate those resources-implications for social service providers and first responders. Violence Vict 27(6):871-894. https://doi.org/10.1891/ 0886-6708.27.6.871

Douglass MD, D'Aguanno S, Jones S (2020) Women as active agents: female perpetrators of sexual harassment and domestic abuse. Evol Behav Sci 14:32-49. https://doi.org/10.1037/ebs0000171 
Drijber BC, Reijnders UJL, Ceelen M (2013) Male victims of domestic violence. J Fam Violence 28(2):173-178. https://doi.org/10.1007/s10896-012-9482-9

Du Mont J, Macdonald S, White M, Turner L (2013) Male victims of adult sexual assault: A descriptive study of survivors' use of sexual assault treatment services. J Interpers Violence 28(13):2676-2694. https://doi.org/10.1177/0886260513487993

Dutton DG (2010) The gender paradigm and the architecture of antiscience. Partn Abus 1:5-25. https://doi.org/10.1891/1946-6560.1.1.5

Dutton DG (2012) The case against the role of gender in intimate partner violence. Aggress Violent Beh 17(1):99-104. https://doi.org/10.1016/j.avb.2011.09.002

Eckstein J, Cherry JN (2015) Perceived characteristics of men abused by female partners: blaming, resulting, blaming-excuses, or normal? Cult Soc Masc 7(2):140-153. https://doi.org/10.3149/ CSM.0702.140

Englar-Carlson M, Kiselica MS (2013) Affirming the strengths in men: a positive masculinity approach to assisting male clients. J Couns Dev 91:399-409. https://doi.org/10.1002/j.15566676.2013.00111.x

Fisher NL, Pina A (2013) An overview of the literature on female-perpetrated adult male sexual victimization. Aggress Violent Beh 18:54-61. https://doi.org/10.1016/j.avb.2012.10.001

Gerson J (2013) Man who ran Canada's only shelter dedicated solely to male victims of domestic abuse dies in apparent suicide. National Post. Retrieved from https://nationalpost.com/news/canada/earlsilverman-who-ran-mens-safe-house-dies-in-apparent-suicide

Hall RE (2012) The feminization of social welfare: Implications of cultural tradition vis-à-vis male victims of domestic violence. J Sociol Soc Welfare 39(3):7-27

Hogan KF, Hegarty JR, Ward T, Dodd LJ (2012) Counsellors' experiences of working with male victims of female-perpetrated domestic abuse. Couns Psychother Res 12(1):44-52. https://doi.org/10.1080/ 14733145.2011.630479

Huntley AL, Potter L, Williamson E, Malpass A, Szilassy E, Feder G (2019) Help-seeking by male victims of domestic violence and abuse (DVA): a systematic review and qualitative evidence synthesis. BMJ Open 9:1-13. https://doi.org/10.1136/bmjopen-2018-021960

Javaid A (2017) 'Walking on egg shells': policing sexual offences against men. Police J Theory Pract Principles 90(3):228-245. https://doi.org/10.1177/0032258X16677357

Laskey P, Bates EA, Taylor JC (2019) A systematic literature review of intimate partner violence victimisation: an inclusive review across gender and sexuality. Aggress Violent Beh 47:1-11. https://doi. org/10.1016/j.avb.2019.02.014

Lysova A, Dim EE (2020) Severity of victimization and formal help seeking among men who experienced intimate partner violence in their ongoing relationships. J Interpers Violence. https://doi.org/ $10.1177 / 0886260520922352$

Lysova A, Dim EE, Dutton D (2019) Prevalence and consequences of intimate partner violence in Canada as measured by the national victimization survey. Partn Abus 10(2):199-221. https://doi.org/10. 1891/1946-6560.10.2.199

Lysova A, Hanson K, Hines DA, Dixon L, Douglas EM, Celi EM (2020a) A qualitative study of the male victims' experiences with the criminal justice response to intimate partner abuse in four Englishspeaking countries. Crim Justice Behav. https://doi.org/10.1177/0093854820927442

Lysova A, Hanson K, Dixon L, Douglas EM, Hines DA, Celi EM (2020b) Internal and external barriers to help seeking: voices of men who experienced abuse in the intimate relationships. Int J Offender Ther Comp Criminol. https://doi.org/10.1177/0306624X20919710

Machado A, Santos A, Graham-Kevan N, Matos M (2017) Exploring help seeking experiences of male victims of female perpetrators of IPV. J Fam Violence 32:513-523. https://doi.org/10.1007/ s10896-016-9853-8

Mankind Initiative (2021) Retrieved from https://www.mankind.org.uk/

McCarrick J, Davis-McCabe C, Hirst-Winthrop S (2016) Men's experiences of the criminal justice system following female perpetrated intimate partner violence. J Fam Violence 31(2):203-213. https:// doi.org/10.1007/s10896-015-9749-z

National Domestic Violence Hotline (2021) Retrieved from https://www.thehotline.org/resources/mencan-be-victims-of-abuse-too/

Neal AM, Edwards KM (2016) Implications of partner agreement of IPV for the gender symmetry controversy. J Fam Violence 31(8):985-987. https://doi.org/10.1007/s10896-016-9866-3

New South Wales Government (2020) I'm a man and experiencing domestic violence. Communities \& Justice. https://www.facs.nsw.gov.au/domestic-violence/my-situation/im-a-man 
New York Post (2017) These shelters help male victims of domestic violence. https://nypost.com/2017/ 10/29/these-shelters-help-male-victims-of-domestic-violence/

Perryman SM, Appleton J (2016) Male victims of domestic abuse: implications for health visiting practice. J Res Nurs 21(5-6):386-414. https://doi.org/10.1177/1744987116653785

Pešáková K (2013) Domestic violence against men in partner relationships-a social work perspective. Czech Slovak Soc Work 13(5):57-63

Ramsey CB (2015) The stereotyped offender: Domestic violence and the failure of intervention. Penn State Law Rev 120(2):337-420

Randle AA, Graham CA (2011) A review of the evidence on the effects of intimate partner violence on men. Psychol Men Masc 12(2):97-111. https://doi.org/10.1037/a0021944

Shorey RC, Sherman AE, Kivisto AJ, Elkins SR, Rhatigan DL, Moore TM (2011) Gender differences in depression and anxiety among male victims of intimate partner violence: the moderating effect of shame proneness. J Interpers Violence 26:1834-1850. https://doi.org/10.1177/0886260510372949

Spencer C, Mallory AB, Cafferky BM, Kimmes JG, Beck AR, Stith SM (2019) Mental health factors and intimate partner violence perpetration and victimization: a meta-analysis. Psychol Violence 9:1-17. https://doi.org/10.1037/vio0000156

Stemple L, Flores A, Meyer IH (2017) Sexual victimization by women: federal data reveal surprising prevalence. Aggress Violent Beh 34:302-311. https://doi.org/10.1016/j.avb.2016.09.007

Straus MA (2011) Gender symmetry and mutuality in perpetration of clinical-level partner violence: empirical evidence and implications for prevention and treatment. Aggress Violent Beh 16(4):279288. https://doi.org/10.1016/j.avb.2011.04.010

Thobejane TD, Luthada V (2019) An investigation into the trend of domestic violence on men: the case of South Africa. OIDA Int J Sustain Dev 12(3):11-18

Tsang WW (2015) Do male victims of intimate partner violence (IPV) deserve help? Some reflections based on a systematic review. Hong-Kong J Soc Work 49(1/2):51-63. https://doi.org/10.1142/S0219 246215000066

Tsui V, Cheung M, Leung P (2010) Help-seeking among male victims of partner abuse: men's hard times. J Community Psychol 38(6):769-780. https://doi.org/10.1002/jcop.20394

Walker A, Lyall K, Silva D, Craigie G, Mayshak R, Costa B, Hyder S, Bentley A (2020) Male victims of female-perpetrated intimate partner violence, help-seeking, and reporting behaviors: a qualitative study. Psychol Men Masc 21(2):213-223. https://doi.org/10.1037/men0000222

World Health Organization (2012) Understanding and addressing violence against women. https://apps. who.int/iris/bitstream/handle/10665/77432/WHO_RHR_12.36_eng.pdf;sequence=1 